Sādhanā Vol. 40, Part 2, April 2015, pp. 351-367. (c) Indian Academy of Sciences

\title{
A fuzzy logic based clustering strategy for improving vehicular ad-hoc network performance
}

\author{
ALI ÇALHAN \\ Computer Engineering Department, Technology Faculty, Duzce University, \\ Duzce 81620, Turkey \\ e-mail: alicalhan@duzce.edu.tr
}

MS received 22 May 2014; revised 30 October 2014; accepted 5 November 2014

\begin{abstract}
This paper aims to improve the clustering of vehicles by using fuzzy logic in Vehicular Ad-Hoc Networks (VANETs) for making the network more robust and scalable. High mobility and scalability are two vital topics to be considered while providing efficient and reliable communication in VANETs. Clustering is of crucial significance in order to cope with the dynamic features of the VANET topologies. Plenty of parameters related to user preferences, network conditions and application requirements such as speed of mobile nodes, distance to cluster head, data rate and signal strength must be evaluated in the cluster head selection process together with the direction parameter for highly dynamic VANET structures. The prominent parameters speed, acceleration, distance and direction information are taken into account as inputs of the proposed cluster head selection algorithm. The simulation results show that developed fuzzy logic (FL) based cluster head selection algorithm (CHSA) has stable performance in various scenarios in VANETs. This study has also shown that the developed $\mathrm{CHSA}_{\mathrm{FL}}$ satisfies well the highly demanding requirements of both low speed and high speed vehicles on two-way multilane highway.
\end{abstract}

Keywords. Vehicular ad-hoc networks; fuzzy logic; clustering.

\section{Introduction}

VANET technology uses moving vehicles as nodes to form a wireless mobile network. It aims to provide fast and cost-efficient data transfer for the advantage of passenger safety and comfort. To improve road safety and travel comfort of voyagers and drivers, Intelligent Transport Systems (ITS) are developed recently. ITS proposes to manage vehicle traffic, support drivers with safety and other information, and provide some services such as automated toll collection and driver assist systems (Karagiannis et al 2011). In essence, VANETs provide new prospects to improve advanced solutions for making reliable communication between vehicles. VANETs can be defined as a part of ITS which aims to make transportation systems faster and smarter in which vehicles are equipped with some short-range and medium-range wireless communication (Booysen et al 2012). In a VANET, wireless vehicles are able to communicate directly with each 
other (i.e., emergency vehicle warning, stationary vehicle warning) and also served various services (i.e., video streaming, internet) from access points (i.e., 3G or 4G) through roadside units (Karagiannis et al 2011).

Generally, two kinds of communications are supposed in VANETs: Vehicle-to-Vehicle (V2V) and Vehicle-to-road side units (or Vehicular-to-Infrastructure, V2I) (Trivedi et al 2011). One of the key objectives of VANETs is to implement ITS for safety travelling and ease of applications. For this reason, an embedded module named On-Board Unit (OBU) is deployed in vehicles for ITS (Hantaksinopas et al 2010). To provide V2V and V2I communications, the OBU consists of a wireless communication unit such as Dedicated Short Range Communication (DSRC) or 3G, a GPS unit, a memory set, and a processor. In figure 1 the VANET's structure is illustrated.

Commonly, VANET architecture services two types of communication devices namely OBU and RSU (Road-Side Unit). As stated earlier, OBUs are installed in vehicles, and RSUs are stationary devices and placed on roadsides. The RSUs act similar to an access point and capable of providing communications with infrastructures (i.e., 2G, 3G, fibre optic, or microwave). The OBU provides travel assistant safety applications (i.e., accident warning systems) between vehicles and also communicate with RSU for getting specific applications from wireless network technologies (i.e., downloads and emails). Also, the data in VANETs are classified into real-time (i.e., video streaming) and nonreal-time (i.e., traffic and weather information) traffics (Su \& Zhang 2007). These applications are also categorized into transport efficiency, safety, and information/entertainment. Each application has different requirements in VANETs. A video

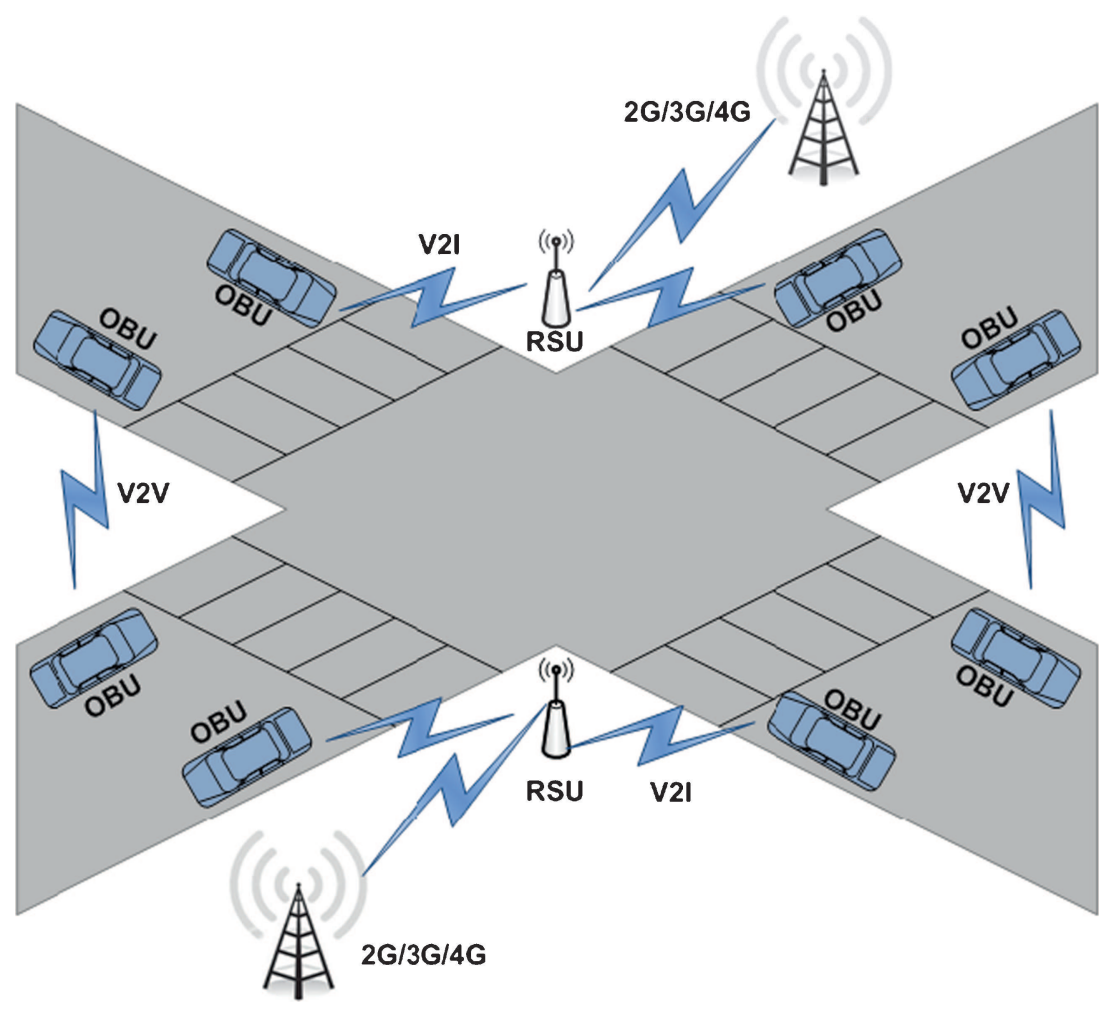

Figure 1. An illustration of VANET structure. 
streaming requires high data rate with reliability and the warning system requires low delay with no loss. To supply these diverse requirements of vehicles and voyagers, the OBUs must be capable of communicating with RSUs and the RSUs must be connected to different communication technologies.

In VANETs, the network topology changes rapidly due to high and fast mobility of vehicles. The rapid changes of VANET topology can break communication links among OBUs and RSUs. So, the communication channels are to be reconstructed between source and destination OBUs and RSUs. In case of changing network conditions, the packet delay and data congestion level increase significantly, and also the number of vehicles in the network varies in time rapidly. For supporting packet delivery in Ad-Hoc manner network structures, conflict-free medium access protocols (i.e., TDMA, FDMA) cannot be advantageous, but the contention based protocols such as CSMA/CA are successfully used instead of these protocols in VANETs.

As mentioned before, two main units, RSU and OBU standards in VANETs have been developed by IEEE which is referred to as Wireless Access in Vehicular Environment (WAVE) (Jiang \& Delgrossi 2008). WAVE consists of IEEE 802.11p and IEEE 1609.X protocols (Urmeneta 2010). Physical and MAC layers of WAVE are dealt with IEEE 802.11p and the upper layers are designed by IEEE 1609.X. RSU and OBU systems use $10 \mathrm{MHz}$ bandwidth in $5.9 \mathrm{GHz}$ band and supported 3 to $27 \mathrm{Mbps}$ data rates depending on the modulation scheme (Maulik \& Vijay 2012). In IEEE 802.11p protocol, CSMA/CA is used for medium access control.

IEEE 802.11p protocol has problems with low throughput, high collision rate, and predictability in high density networks (Hafeez et al 2010). Many researchers have proposed cluster based medium access control protocols to improve the reliability and performance of VANETs. Clustering process is an effective method to reduce data congestion and support QoS over wireless mobile networks that is used to provide fair channel access, decrease channel contention, enhance the network capacity by the spatial reuse of the network resources and effectively control the network structure (Hafeez et al 2012). The most important problem is the overhead presented to select the Cluster Head $(\mathrm{CH})$ in the clustering process and to continue the connection in a fast changing and highly dynamic network topology (i.e., VANETs). The clustering algorithms have been proven to be an effective approach of forming a network into a connected hierarchy of various wireless networks (i.e., Sensor Networks). Sophisticated CH selection algorithms should consider more than one criteria and a methodology to combine and process them. Due to their nonlinearity and generalization capability, artificial intelligence based approaches (i.e., artificial neural networks and fuzzy logic) are mostly used for pattern classifiers (Onel et al 2004; Sun 2007). Fuzzy logic is an efficient multi-attribute decision method since it corresponds to human expert reasoning. In this study, we propose a fuzzy logic based clustering solution to solve the aforementioned problems for VANETs. Fuzzy logic based $\mathrm{CH}$ selection algorithm should initialize selection process considering available vehicles e.g., acceleration, distance, remaining battery, cost, speed, QoS parameters, and so on.

The contributions of this study can be summarized as follows:

- Developed cluster head selection algorithm is modelled using MATLAB software. The network model employing the proposed algorithm and the example scenarios are implemented in OPNET Modeler simulation software. During the simulation, both parts work concurrently for more realistic estimation and substantiation.

- A new multi-criteria $\mathrm{CH}$ selection system, which has the ability to adapt its structure according to the application requirements and network conditions, is proposed. 
- The proposed $\mathrm{CH}$ selection algorithm is different from one of the last studies i.e., (Hafeez et al 2013) due to direction parameter taken into account (for two-way multilane highway) and the $\mathrm{CH}$ selection process aimed with RSUs.

- To the best knowledge of authors, using direction, speed, distance and acceleration parameters for decision making of cluster head in order to optimize $\mathrm{CH}$ selection process is the first time.

The remainder of the paper is organized as follows: Section 2 summarizes some of the related works found in the literature. The developed fuzzy logic based $\mathrm{CH}$ selection approach is discussed in section 3. Section 4 includes the simulation results of case studies. The final section provides the summary about the study with final remarks.

\section{Related works}

There are many clustering algorithms in the literature used in wireless networks especially for Sensor Networks, however, there are only a few researches on clustering techniques about VANETs. In this section, a review of $\mathrm{CH}$ algorithms separately for VANETs was given. A MAC protocol that has clustering based multichannel was proposed for VANETs (Su \& Zhang 2007). The vehicles are equipped with two antennas and they can be activated on diverse channels at the same time. The vehicle that sends firstly a message for inviting the vehicles to join and has more cluster members will be elected as a cluster head. There are three steps proposed for $\mathrm{CH}$ algorithm. Firstly, the Cluster Configuration Protocol sets entirely vehicles in the same direction into clusters, and each cluster contains a $\mathrm{CH}$ vehicle. Secondly, the Intercluster Communication Protocol orders the transmissions of real-time safety messages and nonreal-time traffics between clusters over two distinct IEEE 802.11 MAC-based channels. Thirdly, the Intracluster Coordination-Communication Protocol services multichannel MAC algorithms for each $\mathrm{CH}$ vehicle to manage the some main tasks within its specific cluster. As cluster members move frequently in and out of the cluster boundary, this algorithm results in high frequent cluster topology variations. Also, it has a very high cost and needs very strict syncronization between all vehicles.

A mobility based clustering method utilizing the affinity propagation algorithm was proposed (Shea et al 2009). The algorithm finds clusters that decrease the relative mobility and distance from each $\mathrm{CH}$ to its cluster members. The nearest vehicle to its neighbours will be elected as a cluster head. The authors proposed a dynamic and distributed cluster head election criteria to form the network into clusters dynamically with a learning scheme for predicting the future speed and position of all cluster members using a fuzzy logic inference system (Hafeez et al 2012). The $\mathrm{CH}$ algorithm was based on vehicles' distance from other vehicles within their neighborhood and their relative speed. The authors aimed to solve the problems which were encountered aforementioned studies in the literature with using fuzzy logic based $\mathrm{CH}$ selection algorithm (Hafeez et al 2012). It is assumed that vehicles are moving in a one-way multilane highway. The authors introduce a stable multi-hop clustering method (Ucar et al 2013). The scenarios consists of a two line and two way road and cluster head duration, average cluster member duration and cluster head change parameters were considered. But, there is no information about physical and MAC layer of VANET. The mobility model in Ahizoune \& Haid (2012) is the freeway mobility model with four highway lanes and all the lanes are in the same direction. Mobility, number of neighbours, and leadership duration are considered in clustering algorithm. Maglaras \& Katsaros (2012) have only considered V2V communication between vehicles. A distributed 
clustering algorithm which forms stable clusters based on force directed algorithms was proposed and a mobility metric based on forces applied between nodes according to their current and their future position and their relative mobility.

In this study, an alternative approach using fuzzy logic under dynamic network conditions is given. A novel $\mathrm{CH}$ selection algorithm by evaluating direction, speed, acceleration and distance parameters from vehicles in two-way multilane highway is proposed. The developed $\mathrm{CH}$ selection algorithm is modelled using MATLAB software. The VANET model utilizing the proposed algorithm and the example scenarios are executed in OPNET Modeler. Both parts work concurrently during the simulation for more realistic substantiation and estimation.

\section{A new fuzzy logic based clustering head selection algorithm (CHSAFL)}

One of the many problems in VANETs is the dynamic and dense network topology therefore, it causes important routing problems and data congestions. The clustering algorithms have been proven to be an effective approach of forming a network into a connected hierarchy. A network can be transformed smaller and more stable networks by using clustering techniques in dynamic networks such as VANETs. By clustering techniques, the vehicles are grouped into small networks in terms of mobility, distance, direction, etc. (Ahizoune et al 2010; 2012). Figure 2 shows an example of clustering in VANETs.

Stable clustering techniques can reduce the overhead of reclustering and provide an efficient hierarchical network topology. During the election of CHs in VANETs, cluster head candidates select one candidate to be the $\mathrm{CH}$. Fewer $\mathrm{CH}$ changes provide a more stable cluster. For this purpose, cluster candidates must choose a candidate that has the potential to be a $\mathrm{CH}$ longer than other cluster head candidates. Each vehicle is able to communicate with its $\mathrm{CH}$ directly and the vehicles are able to communicate with the others either directly or, via their $\mathrm{CH}$.

Cluster stability is a significant objective in VANETs as a performance criteria of a $\mathrm{CH}$ selection algorithm and can be defined as the number of $\mathrm{CH}$ variations and number of a vehicle changing its cluster head. With the optimum $\mathrm{CH}$ selection process, the cluster stability can be intensely improved. Many clustering approaches have been developed and these algorithms

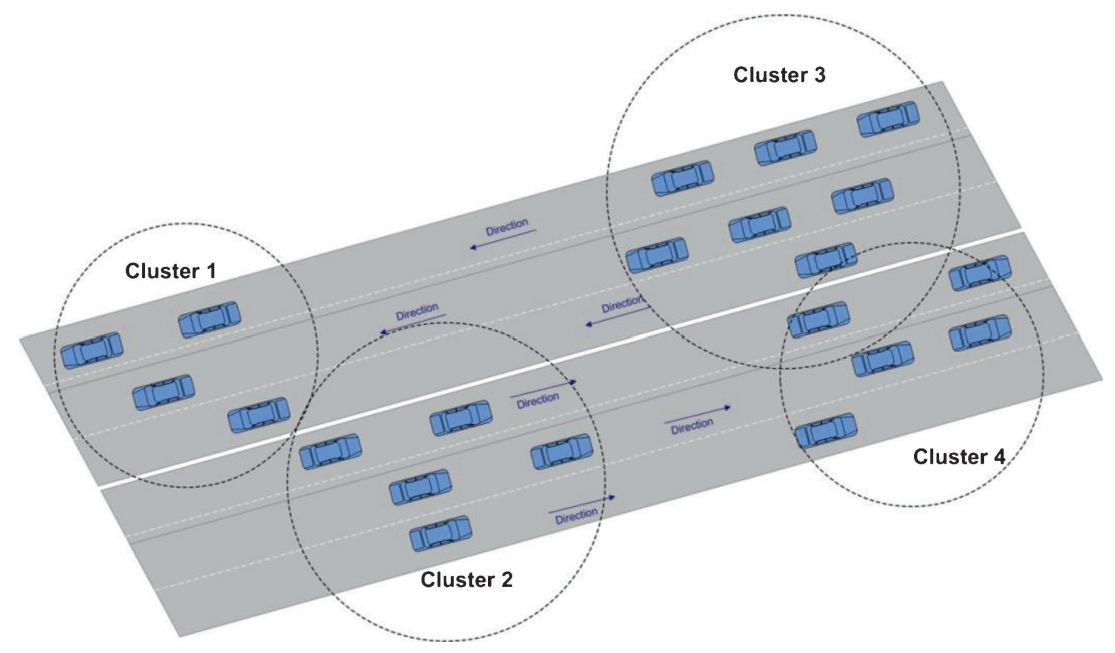

Figure 2. Clusters in VANETs. 
can be categorized in two main topics. The first one is location service dependant and utilizes parameters such as speed, location, direction, etc. The second one uses various parameters such as vehicle density, connectivity, radio propagation, etc. (Vodopivec et al 2012). Both clustering methods are based on mathematically measurable parameters and due to these parameters, the $\mathrm{CH}$ selection process must be performed with a multi-attribute decision making (MADM) method in VANETs. As mentioned before, fuzzy logic is an efficient multi-attribute decision method since it corresponds to human expert reasoning. This method is applied to clustering algorithms as it overcomes radio environment fluctuations and uncertainty as well as the intersystem parameter heterogeneity such as shadowing, measurements averaging, traffic model variations. Consequently, it is vital to make automatically adapt the fuzzy logic control to all variations. Therefore, in the proposed $\mathrm{CH}$ selection system a fuzzy logic-based approach has been adopted. Accordingly, in this study, we propose a fuzzy logicbased $\mathrm{CH}$ selection algorithm which considers the parameters; direction, speed, acceleration and distance as inputs in order to handle any $\mathrm{CH}$ selection process. The block diagram of the proposed $\mathrm{CH}$ selection system is given in figure 3.

Figure 3 illustrates the essential components of the fuzzy based $\mathrm{CH}$ selection system. The first phase of the $\mathrm{CH}$ selection system is to give the measured parameters into a fuzzifier. The task of the fuzzifier is transforming the actual quantities into fuzzy sets. For instance, if the acceleration is considered in crisp set, it can be represented as decelerate, same or accelerate in the corresponding fuzzy set. The membership values $(\mu)$ are generated by mapping the values obtained for a specific parameter into a membership function. Then, the fuzzy conversions are performed by utilizing a reverse engine (i.e., defuzzifier) to produce output. In the last phase, the calculated output is exploited for choosing the best candidate $\mathrm{CH}$.

The clustering approach promises the scalability of networks, where high mobility of the replacing vehicles within the networks causes a number of difficulties. For performance evaluations of cluster head selection approaches common performance metrics are utilized such as $\mathrm{CH}$ changes, $\mathrm{CH}$ stability, etc. But, reasonable comparison of various clustering approaches is a hard task due to lack of scenarios and standard testing processes, therefore standardization and more researches are needed in this subject (Vodopivec et al 2012).

The re-clustering procedure is executed periodically, which generally leads to the less stable cluster structure. Clustering procedure can be difficult to apply any Mobile Ad-Hoc networks,

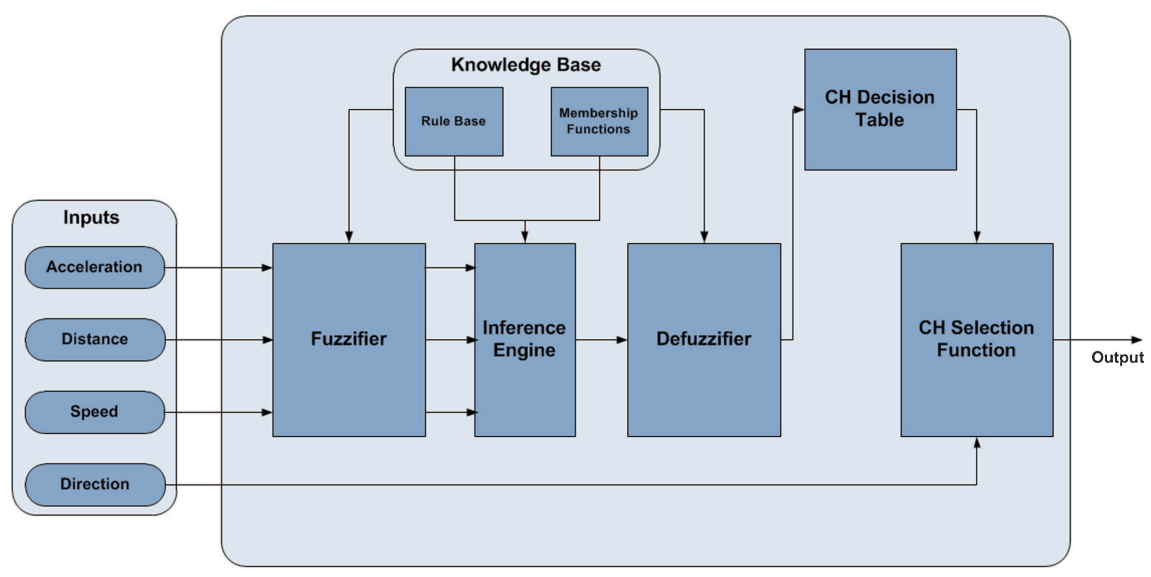

Figure 3. Block diagram of the proposed fuzzy logic based $\mathrm{CH}$ selection system. 


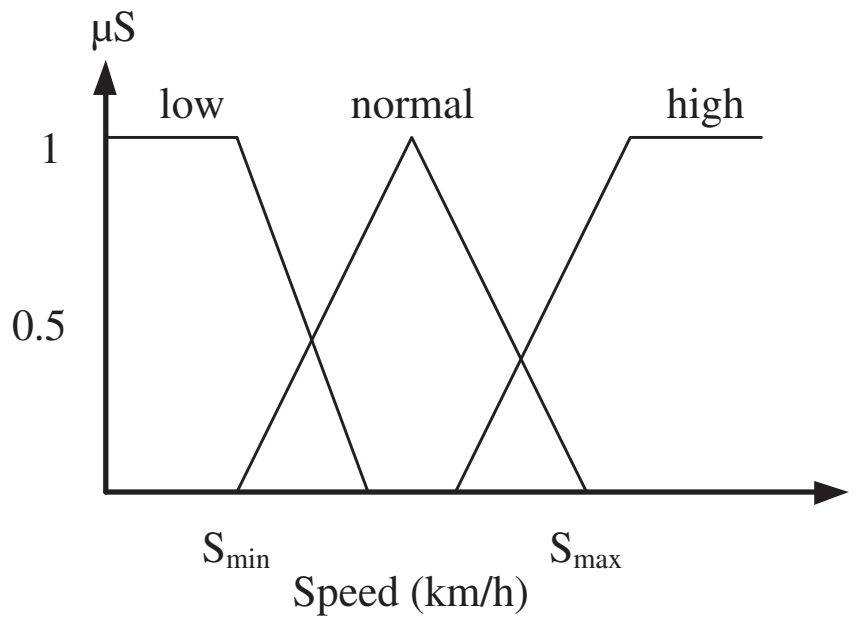

Figure 4. Fuzzy membership functions for speed (S).

but in VANETs, there are several advantages of applying clustering; firstly, the network topology is constrained by highways and then secondly, the vehicles are moving in groups naturally on roads. These benefits give a chance to develop the clustering algorithms for VANETs.

In the proposed fuzzy logic based clustering algorithm, membership functions of the fuzzy logic scheme inputs are shown in Figures 4, 5 and 6, respectively. Trim and trapezoid are preferred as the fuzzy membership functions owing to their capability of achieving better performance for especially real time applications (Çeken et al 2010; Wang 1994).

Our fuzzy logic system has totally 27 fuzzy rules and some developed fuzzy rules are illustrated in table 1.

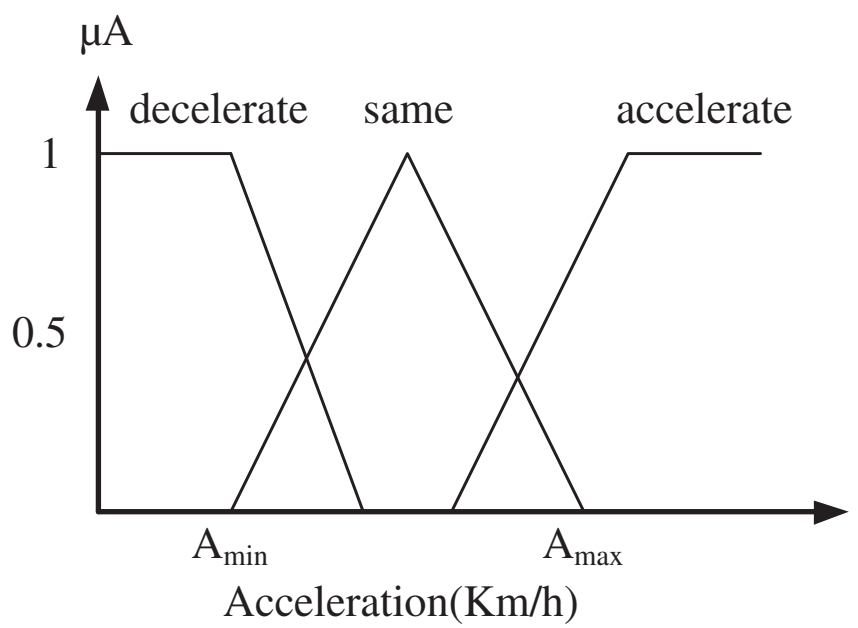

Figure 5. Fuzzy membership functions for acceleration (A). 


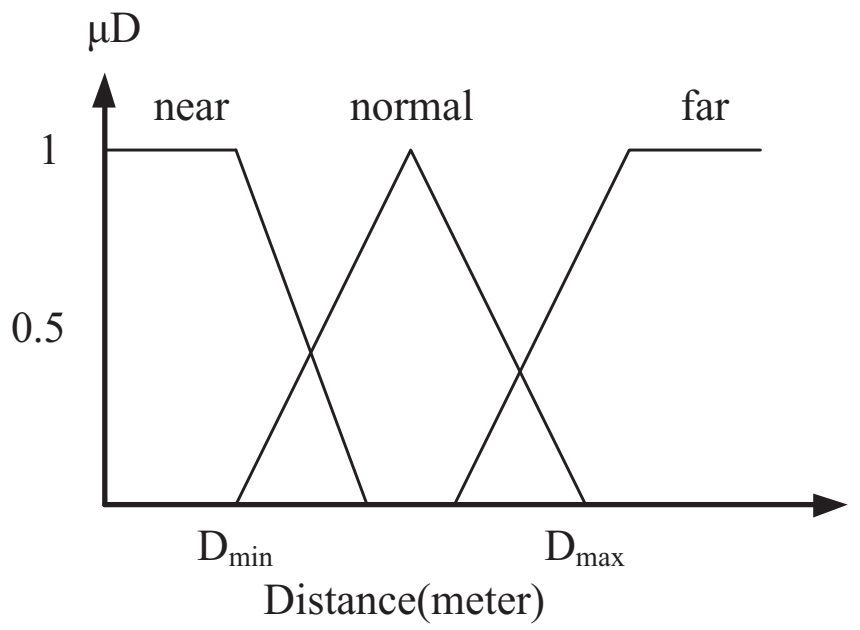

Figure 6. Fuzzy membership functions for distance (D).

The candidate values of vehicles is considered to vary between and 10 where represents the weakest, whereas 10 denotes the strongest candidacy level of quantification in this study. The analytic model of the fuzzy inference system is as follows. Three-dimensional pattern vector (input of the fuzzifier) for candidate access points is:

$$
P V_{C}=\left[S_{C} ; A_{C} ; D_{C}\right]
$$

where $\mathrm{S}$ is speed, $\mathrm{A}$ is acceleration, and $\mathrm{D}$ is the Distance value of vehicle. Three-dimensional fuzzy pattern vectors (output of fuzzifier and input of inference engine) for candidate cluster head is:

$$
P V_{F}=\left[P F_{1} ; P F_{2} ; P F_{3}\right]
$$

Since the product inference rule is utilized in the fuzzy inference engine, then, for a new pattern vector, the contribution of each rule in the fuzzy rule base is computed by:

$$
C_{r}=\prod_{i=1}^{3} \mu_{F_{i}}\left(P_{i}\right) .
$$

Table 1. Example of fuzzy rules.

IF (Speed is Low) and (Acceleration is Decelerate) and (Distance is Near) then (Output is 3)

IF (Speed is High) and (Acceleration is Decelerate) and (Distance is Near) then (Output is 7)

IF (Speed is Normal) and (Acceleration is Same) and (Distance is Near) then (Output is 10)

IF (Speed is Normal) and (Acceleration is Decelerate) and (Distance is Normal) then (Output is 5)

IF (Speed is High) and (Acceleration is Same) and (Distance is Normal) then (Output is 3)

IF (Speed is High) and (Acceleration is Accelerate) and (Distance is Far) then (Output is 0.5) 
Since we have 27 rules and a center average defuzzifier is utilized, the output of the defuzzifier is:

$$
M_{a}=\frac{\sum_{l=1}^{27} y^{l}\left(\prod_{i=1}^{3} \mu_{F_{i}}\left(P_{i}\right)\right)}{\sum_{l=1}^{27}\left(\prod_{i=1}^{3} \mu_{F_{i}}\left(P_{i}\right)\right)}
$$

where, $\mathrm{y}^{1}$ is the output of the rule 1 .

The proposed scheme mainly consists of two main sections. First, the cluster configuration section decides which cluster the vehicles will be joined, because a vehicle can participate in any cluster in both directions. A vehicle must be in only one cluster at the same time. So, in the proposed simulations, each vehicle in an RSU coverage area takes a cluster affinity value in an information packet from the RSU. This value indicates the vehicles' affinity levels of a cluster which is proportional to the distance from RSU. A vehicle can join a cluster owing to the affinity level that defines the proximity of the vehicle to RSU. For example, if a vehicle is in the coverage area of two RSUs, one of them is $100 \mathrm{~m}$ away from the vehicle, and the other is $300 \mathrm{~m}$ away from the vehicle, finally, the affinity level of the first cluster is bigger than the second one. Therefore, the vehicle decides to join to the clusters in the first RSU's coverage area according to affinity level. RSSI (Received Signal Strength Indicator) values of RSUs are used for affinity level.

In the second section, the cluster head selection is presented. All of the vehicles in a cluster send to each other an information packet that includes its own speed, distance, acceleration, and distance parameters. Each vehicle extracts the related parameters in order to use as inputs of the proposed $\mathrm{CH}$ selection algorithm. The vehicles determine $\mathrm{CH}$ candidacy levels using its fuzzy inference system with those parameters as shown in table 1 . The candidacy level of each vehicle is compared with that of current $\mathrm{CH}$. If the difference between the compared values is greater than the current $\mathrm{CH}$, the new vehicle is selected as a $\mathrm{CH}$. $\mathrm{CH}$ candidate value is a real number that varies between 0 and 10 where 0 indicates the weakest, whereas 10 represents the strongest candidacy level of quantification. For instance; when a determined vehicle has a $100 \mathrm{~km} / \mathrm{h}$ speed, $60 \mathrm{~m}$ distance to the $\mathrm{CH}$, and zero acceleration, then the candidacy of this vehicle is produced as 6.0471 by the proposed fuzzy logic algorithm. The vehicle which has the maximum candidacy level is selected as a cluster head.

The sequence diagram of the proposed fuzzy logic based $\mathrm{CH}$ selection algorithm is outlined in figure 7. In the proposed simulation model, the direction parameter of vehicles also affects the $\mathrm{CH}$ selection process. The vehicles are grouped into clusters which have the same directions in the second section of the proposed $\mathrm{CH}$ selection scheme. A vehicle does not participate a cluster whose $\mathrm{CH}$ moves in its opposite direction. The $\mathrm{CHs}$ can communicate with each other even if they are in different directions. According to the vehicles' parameters in each cluster, the FIS produces an output that defines the $\mathrm{CH}$ candidacy level of vehicles and varies from zero to ten. Cluster size varies from one cluster to another according to the vehicles that inside the RSU's coverage area.

The proposed OBU unit is developed using OPNET Modeler software and its cross layer design is outlined in figure 8. It includes physical, MAC, and some upper-layer functions. It is composed of a CSMA/CA based MAC module for communicating with other OBUs and RSUs and fuzzy logic unit which is in charge of managing all of the $\mathrm{CH}$ selection operations. 


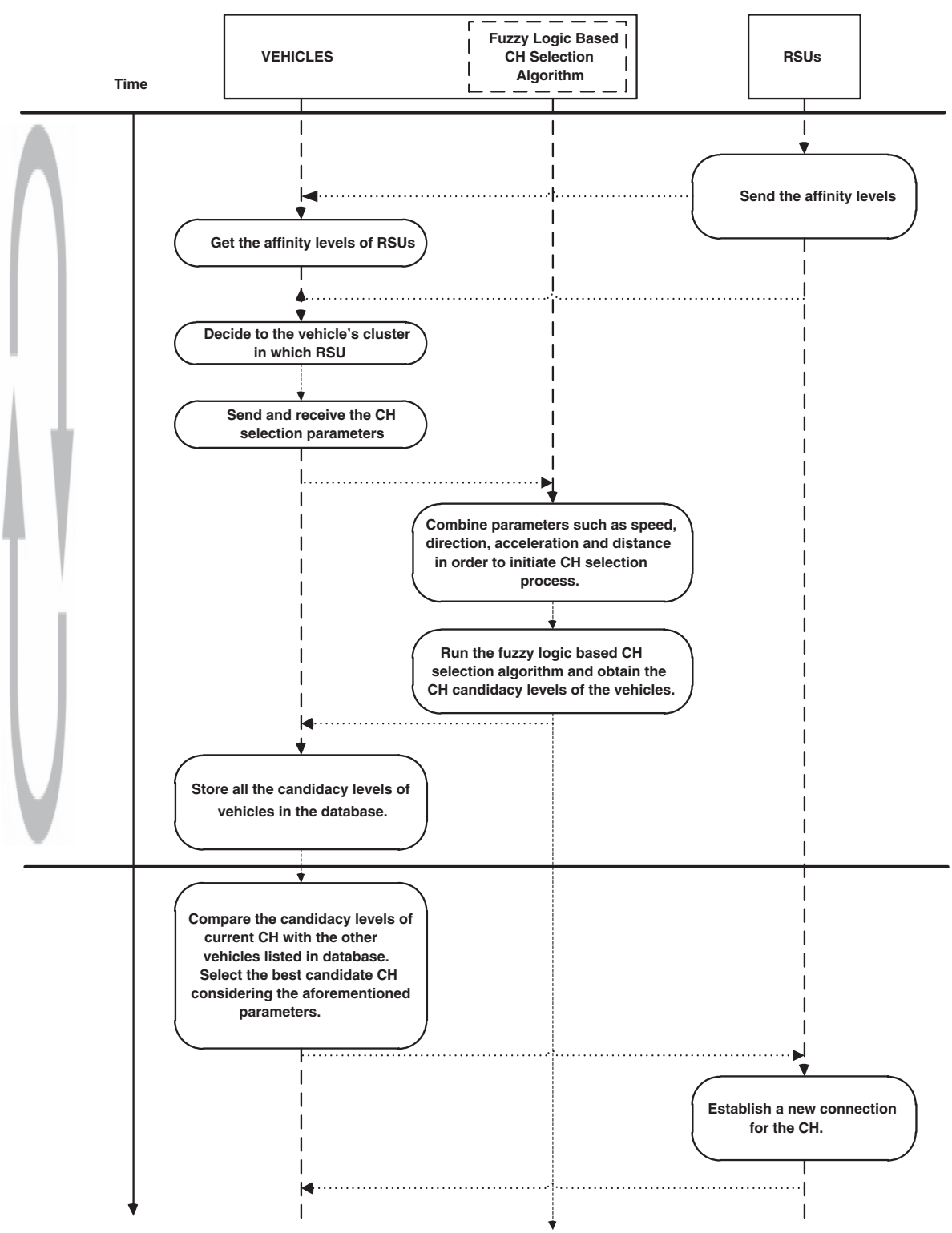

Figure 7. Sequence diagram of the proposed fuzzy logic based $\mathrm{CH}$ selection system.

\section{Simulation models and performance analysis of the proposed CHSAFL}

The simulation models and scenarios are developed using OPNET Modeler for more accurate and realistic performance evaluation. The execution of the fuzzy logic based $\mathrm{CH}$ selection operation is executed with the MATLAB Fuzzy Inference System editor. Both MATLAB and OPNET work together for more sensible substantiation and estimation during the simulation. The proposed VANET architecture consists of vehicles equipped with OBUs, RSUs, and various wireless 


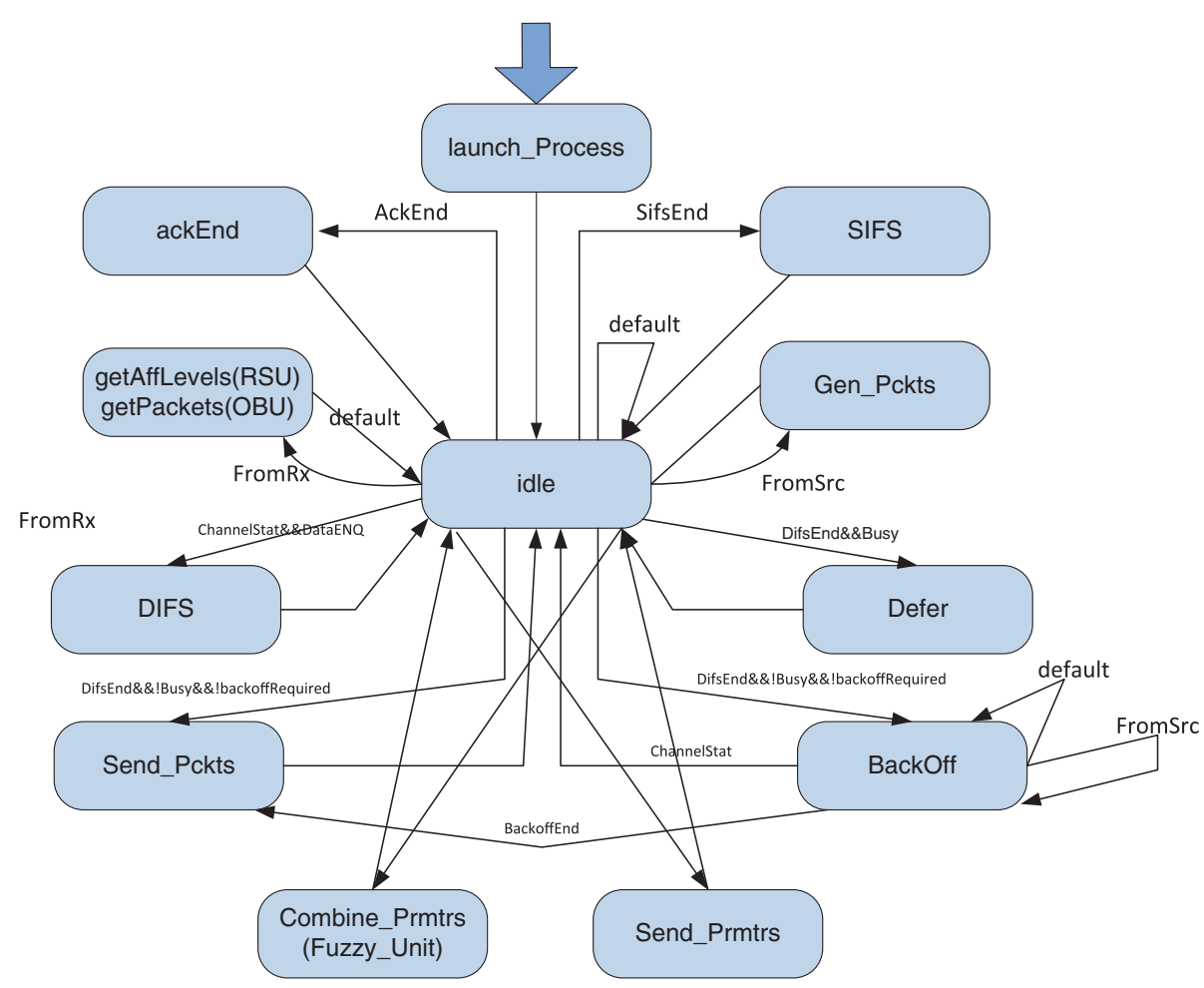

Figure 8. The OBU cross-layer process model.

base stations $(2 \mathrm{G} / 3 \mathrm{G} / 4 \mathrm{G})$ in our simulation scenario, illustrated in figure 9. Both the vehicle-tovehicle and vehicle-to-RSU communications are considered in this study. The coverage area of RSUs and vehicles are at about 1000 and $300 \mathrm{~m}$, respectively. In the simulation scenarios, each vehicle is aware of its location by using positioning services, such as GPS (Global Positioning System). This enables the position of the vehicle, direction prediction and its speed.

The proposed algorithm is explained with the following scenarios. The other simulation parameters used are given in table 2.

\subsection{Case study 1}

The case studies consist of a $5 \mathrm{~km}$ highway with six lanes, three of each direction, 5 RSUs with $1000 \mathrm{~m}$ coverage area and vehicles as shown in figure 10.

The vehicles move with a constant or variable speed ranging from 30 to $100 \mathrm{~km} / \mathrm{h}$ according to trajectory attribute of mobile terminals in OPNET. The RSUs are placed every $1 \mathrm{~km}$. Each RSU sends an information packet every ten milliseconds to the vehicles which are in its coverage area during the simulation runtime. The information packets synchronize the vehicles according to the directions of the vehicles. Firstly, the vehicles are grouped into two clusters in each RSU coverage area according to the directions as illustrated in figure 10. A number of clusters can be in a RSU's coverage area. Secondly, the fuzzy based CH selection algorithm runs on the vehicles' OBUs. Each vehicle sends its own information packets to other vehicles with aforementioned parameters. Direction parameter of the vehicles directly affects the $\mathrm{CH}$ selection process. This 


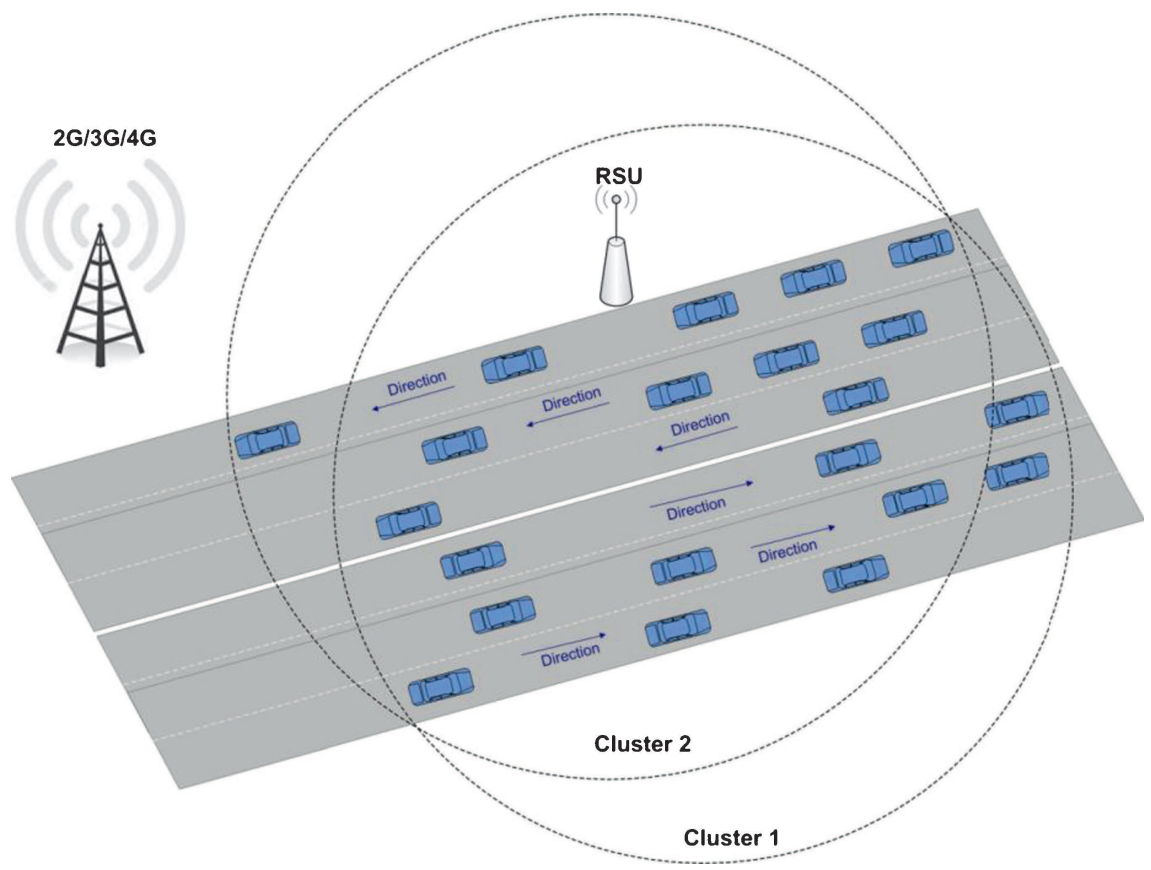

Figure 9. Proposed VANET architecture.

parameter decides to join to the cluster in which the vehicle, and then the proposed fuzzy logic based $\mathrm{CH}$ selection algorithm is performed. In order to show the detailed performance of the proposed algorithm, it is assumed that there are several vehicles in the coverage area of RSU-1 with the same direction named Vec-1, Vec-2 and so on. Eight vehicles are grouped into a cluster by the first section of the proposed fuzzy algorithm with the same direction. Then, each of these vehicles sends the information packets including speed, acceleration and distance parameters to each other. Finally, the parameters are evaluated in the fuzzy logic based CH selection algorithm and the outputs of the algorithm are stored in a database. The output of the fuzzy logic based algorithm is defined as the $\mathrm{CH}$ candidate value of each vehicle. As soon as the fuzzy logic based $\mathrm{CH}$ selection algorithm is performed, each vehicle compares the $\mathrm{CH}$ candidate values of the current vehicle with the ones added to the database, respectively. The vehicle which

Table 2. Simulation parameters.

\begin{tabular}{ll}
\hline MAC Protocol & CSMA/CA Based \\
\hline Frequency & $5.9 \mathrm{GHz}$ \\
Modulation type & QPSK \\
RSU transmitting range & $1000 \mathrm{~m}$ \\
OBU transmitting range & $300 \mathrm{~m}$ \\
Channel bandwidth & $10 \mathrm{MHz}$ \\
Simulation area (for each RSU) & $1000 \mathrm{~m} \times 60 \mathrm{~m}$ \\
Simulation time & $40 \mathrm{sec}$ \\
Vehicles speed & $30-120 \mathrm{~km} / \mathrm{h}$ \\
\hline
\end{tabular}




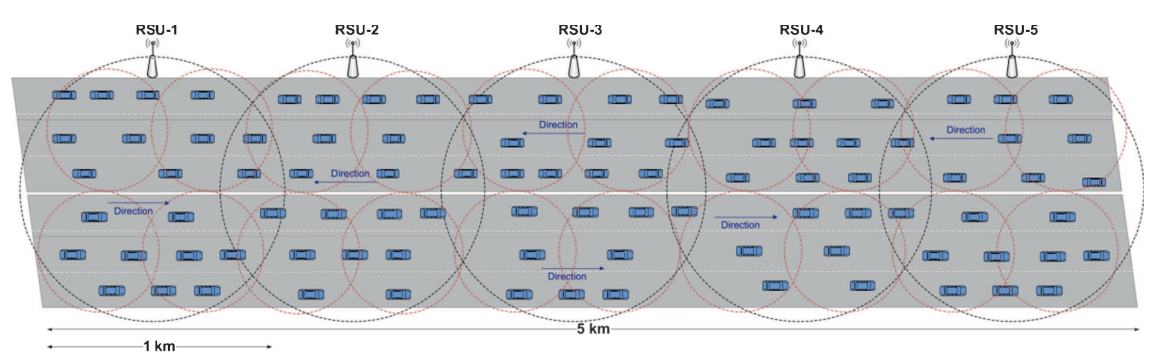

Figure 10. VANET architecture in case studies.

has the maximum candidacy level is selected as a cluster head as mentioned in the previous section.

In the first case study, when the simulation begins, the fuzzy membership functions of speed parameters are reconstructed by the mean velocity of the vehicles in each cluster. Each vehicle named Vec-1, Vec-2 and others have a constant 70, 90, 75, 80, 85, 95, 75, and $100 \mathrm{~km} / \mathrm{h}$ speeds, respectively. The mean velocity can be calculated as $83.75 \mathrm{~km} / \mathrm{h}$ in the scenario. So, according to the mean velocity, Vec-4 and Vec-5 can be selected as a $\mathrm{CH}$. With the other aforementioned parameters such as distance, the Vec-5 is selected as $\mathrm{CH}$ vehicle due to the proposed fuzzy logic based $\mathrm{CH}$ selection algorithm. The parameters are sent and received by the vehicles and the $\mathrm{CH}$ selection operation runs every second. The distance between $\mathrm{CH}$ and vehicles are changed as well as the speeds of the vehicles are constant. During the simulation time, the vehicles' $\mathrm{CH}$ candidate values are illustrated in figure 11 . The simulation results are given only for the vehicles in the RSU-1 coverage area.

\subsection{Case study 2}

In the second case study, all of the simulation parameters and working conditions are the same with the first case study except the variable speeds of the vehicles. To indicate the effects of variable speeds on the proposed algorithm, case study 2 is simulated. At the beginning of the simulation, the vehicles named Vec-1, Vec-2 and others have a 70, 90, 75, 80, 85, 95, 75, and

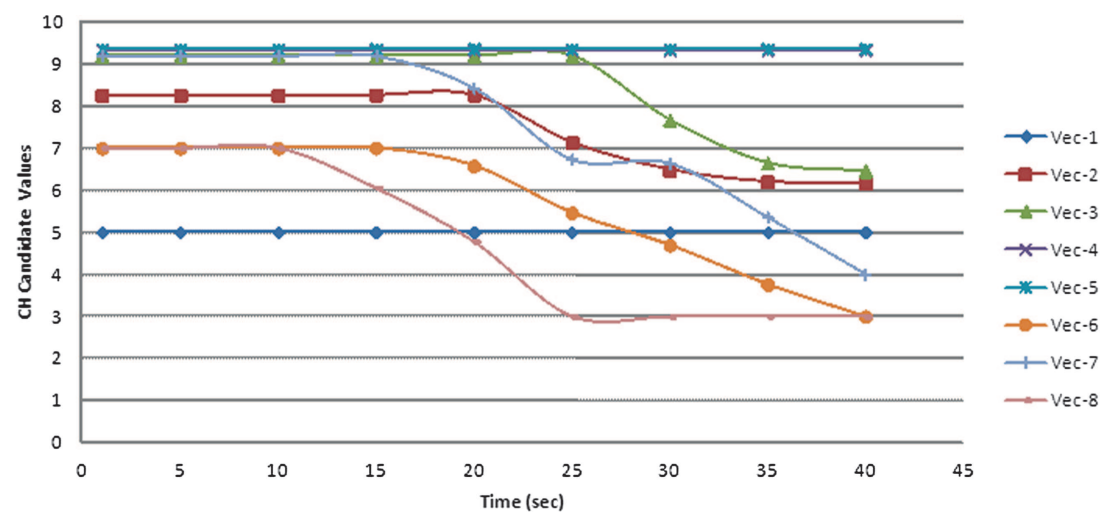

Figure 11. CH candidacy levels of vehicles in case study 1 . 


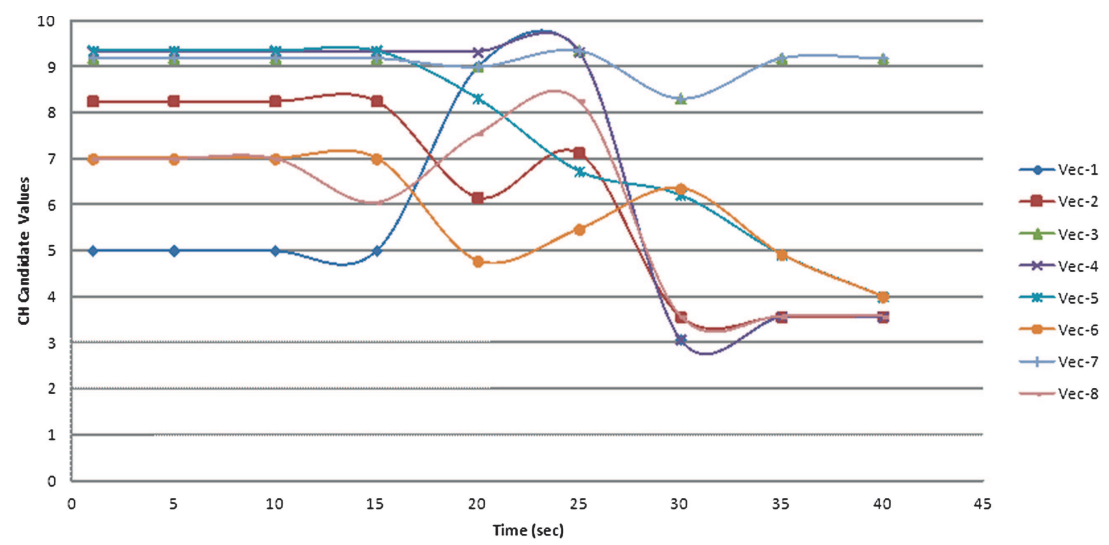

Figure 12. CH candidacy levels of vehicles in case study 2.

$100 \mathrm{~km} / \mathrm{h}$ speeds, respectively for fifteen seconds. So, Vec-5 is selected as the $\mathrm{CH}$ vehicle as in the case study one. During the simulation, some of the vehicles accelerate, while the others decelerate. And a few of the vehicles can stay at the same speed.

At the 16th second, Vec-1, Vec-2 and others change its speed to 80, 90, 85, 80, 75, 95, 85, and $90 \mathrm{~km} / \mathrm{h}$, respectively. Then, new speed values reconstruct the fuzzy membership function of the speed parameter. So, the proposed $\mathrm{CH}$ selection algorithm is performed with speed, acceleration, and distance to $\mathrm{CH}$ parameters. For example, Vec-1 has $80 \mathrm{~km} / \mathrm{h}$ speed, 0.1 acceleration, and $22.2 \mathrm{~m}$ distance to Vec-5 (current $\mathrm{CH}$ ), and then the new candidacy level of Vec-1 is calculated as 9.00. The candidacy level calculation process is performed for each vehicle. Finally, Vec-4 is selected as a $\mathrm{CH}$ which has 9.32 candidate value. For every five seconds to the end of the simulation, with the variable speed, acceleration and distance values, Vec-5, Vec-4, and Vec-3 are selected as $\mathrm{CH}$ due to the proposed fuzzy logic based $\mathrm{CH}$ selection method as presented in figure 12.

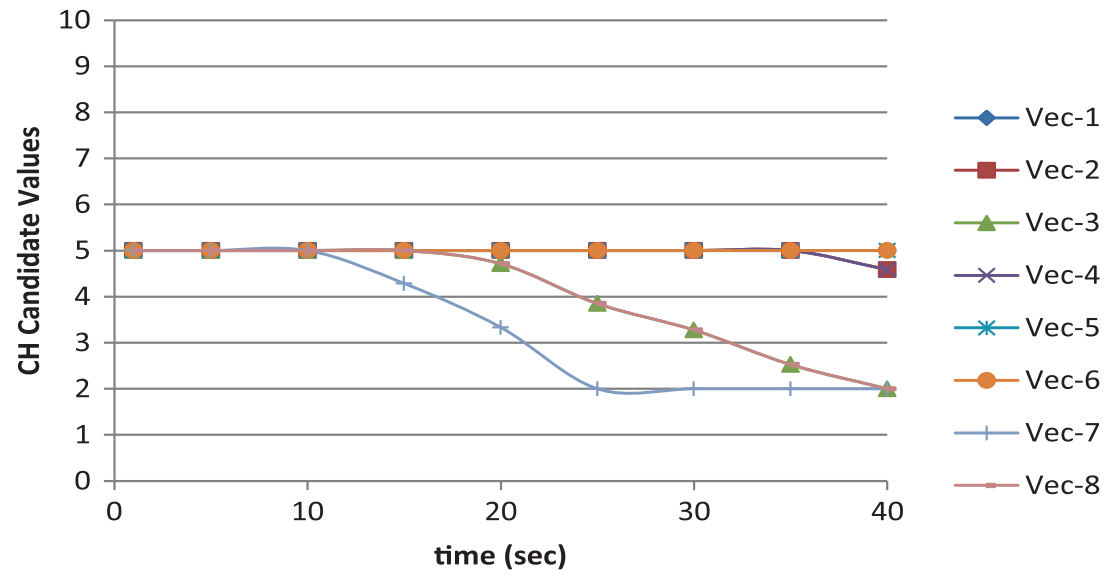

Figure 13. CH candidacy levels of vehicles in case study 3 . 


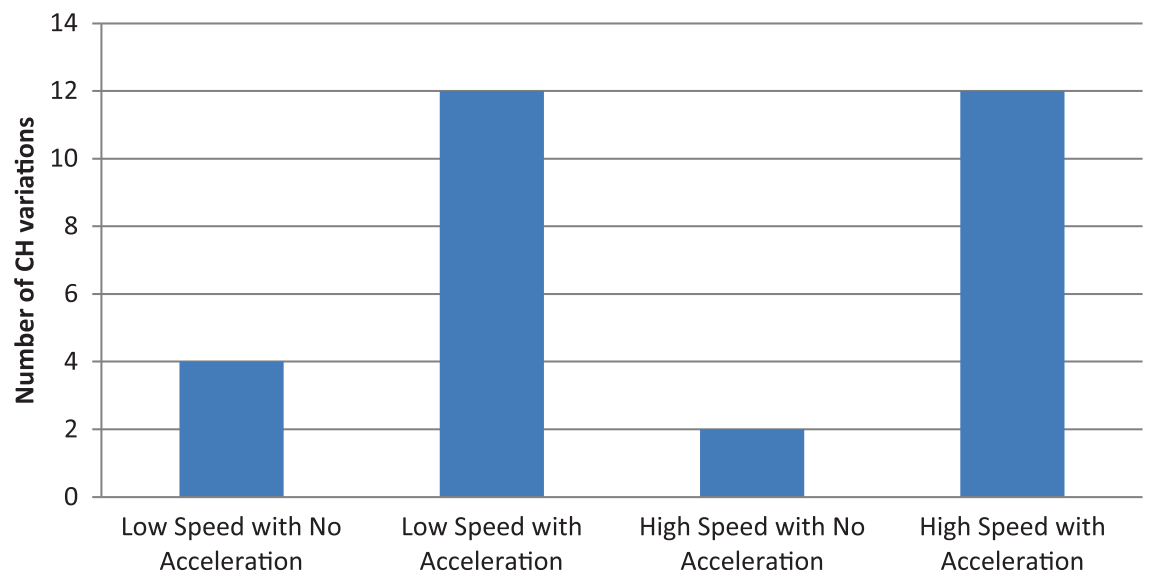

Figure 14. Number of $\mathrm{CH}$ variations.

\subsection{Case study 3}

In the third case study, all of the simulation parameters and working conditions are the same with the first case study except the low speeds of the vehicles. The speeds of the vehicles in the simulations are limited between 15 and $30 \mathrm{~km} / \mathrm{h}$ for the urban areas with heavy traffics. Each vehicle named Vec-1, Vec-2 and others have a constant 15, 20, 25, 20, 15, 15, 30, and $25 \mathrm{~km} / \mathrm{h}$ speeds. Because of the low speeds and heavy traffics, the distances between vehicles are not so much as vehicles in other case studies. So, any vehicles can be selected as cluster head in the third case study. The first vehicle which takes the maximum $\mathrm{CH}$ candidate values is chosen as $\mathrm{CH}$. Vec-1 is selected as $\mathrm{CH}$ in the third case study during the simulation time, the vehicles' $\mathrm{CH}$ candidate values are illustrated in figure 13 .

\subsection{Case study 4}

In the fourth case study, all of the simulation parameters and working conditions are the same with the three case studies. The speeds of the vehicles in the simulations are limited between 30 and $50 \mathrm{~km} / \mathrm{h}$ for the first part of the scenario and in the second part of the simulation scenario, the speeds vary between 90 and $120 \mathrm{~km} / \mathrm{h}$ in this case study. This case study is proposed to show the developed $\mathrm{CH}$ selection algorithm performance for low and high speed vehicles' clusters with acceleration effect. The number of $\mathrm{CH}$ variations of developed algorithm for low and high speed vehicles are illustrated in figure 14. As can be seen in figure 14, low speed and high speed performance evaluations of the developed algorithm are nearly same that shows the stability of the algorithm.

\section{Conclusions}

Cluster head selection process is one of the major challenges in Vehicular Ad-Hoc Networks since there are various parameters must be considered and it is a crucial issue that the algorithm satisfies all vehicles which have diverse speeds. A fuzzy logic based cluster head selection algorithm is proposed which is able to combine direction, speed, acceleration, and distance 
parameters in order to select the most appropriate vehicle as cluster head in this study. The simulation results show that the proposed algorithm can select the vehicle as cluster head with the optimal parameters in case studies, and also, the proposed algorithm satisfies both low speed and high speed vehicles on two-way multilane highway.

\section{References}

Ahizoune A and Hafid A 2012 A new stability based clustering algorithm (SBCA) for VANETs, Local Computer Networks Workshops (LCN Workshops). 2012 IEEE 37th Conference 843(847): 22-25

Ahizoune A, Hafid A and Ben Ali R 2010 A Contention Free Broadcast Protocol for Periodic Safety Messages in Vehicular Ad-Hoc Networks, 35th Annual IEEE Conference on Local Computer Networks and Workshops, (LCN), 48-55

Booysen M J, Zeadally S and Van Rooyen G-J 2012 Performance comparison of media access control protocols for vehicular ad hoc networks. Networks, IET 1(1): 10,19

Çeken C, Yarkan S and Arslan H 2010 Interference aware vertical handoff decision algorithm for quality of service support in wireless heterogeneous networks. Computer Networks 54(5): 726-740

Hantaksinopas I, Oothongsap P and Prayote A 2010 Framework for network selection transparency on vehicular networks, Electrical Engineering/Electronics Computer Telecommunications and Information Technology (ECTI-CON), 2010 International Conference, 593,597, 19-21 May 2010

Hafeez K A, Zhao L, Liao Z and Ma B 2010 Performance analysis of broadcast messages in VANETs safety applications. Proc. IEEE Globecomm Conf. 1-5

Hafeez K A, Zhao L, Liao Z and Ma B N -W 2012 A fuzzy logic based cluster head selection algorithm in VANETs Communications (ICC), 2012 IEEE International Conference 203,207, 10-15 June 2012

Hafeez K A, Zhao L, Mark J W, Shen X and Niu Z 2013 Distributed Multichannel and Mobility-Aware Cluster-Based MAC Protocol for Vehicular Ad Hoc Networks. IEEE Trans. On Vech. Tech. 62(8): 38863902

Jiang D and Delgrossi L 2008 IEEE 802.11p: Towards an international standard for wireless access in vehicular environments, Vehicular Technology Conference, 2008. VTC Spring 2008. IEEE 2036(2040): $11-14$

Karagiannis G, Altintas O, Ekici E, Heijenk G, Jarupan B, Lin K and Weil T 2011 Vehicular networking: A Survey and tutorial on requirements, architectures, challenges, standards and solutions. Communications Surveys \& Tutorials. IEEE 13(4): 584,616 Fourth Quarter 2011

Maulik P and Vijay U 2012 Optimized handoff process in IEEE802.11p based VANET, Emerging Technology Trends in Electronics, Communication and Networking (ET2ECN). 2012 1st International Conference 1(5): 19-21

Maglaras L A and Katsaros D 2012 Distributed clustering in vehicular networks, Wireless and Mobile Computing, Networking and Communications (WiMob). 2012 IEEE 8th International Conference 593(599): $8-10$

Onel T, Ersoy C, Cayırcı E and Parr G 2004 A multicriteria handoff decision scheme for the next generation tactical communications systems. Computer Networks 46(5): 695-708

Su H and Zhang X 2007 Clustering-Based Multichannel MAC Protocols for QoS Provisionings Over Vehicular Ad Hoc Networks, Vehicular Technology. IEEE Transactions 56(6): 3309-3323

Sun J Z 2007 A review of vertical handoff algorithms for cross-domain mobility, in: Proceedings of the International Conference on Wireless Communications. Networking and Mobile Computing (WiCom 2007), Sept. 21-25, 2007, 3156-3159

Su H and Zhang X 2007 Clustering based multichannel MAC protocols for QoS provisionings over vehicular ad hoc networks. IEEE Trans. Vehicular Tech 56(6): 3309-3323

Shea C, Hassanabadi B and Valaee S 2009 Mobility-based clustering in VANETs using affinity propagation, Proc. IEEE Globecomm Conf., pp. 1-6 
Trivedi H, Veeraraghavan P, Loke S, Desai A and Singh J 2011 SmartVANET: The Case for a cross-layer vehicular network architecture, Advanced Information Networking and Applications (WAINA). 2011 IEEE Workshops of International Conference 362(368): 22-25

Urmeneta P 2010 Simulation and improvement of the handover process in IEEE 802.11p based VANETs (Vehicle Ad-Hoc Networks), College of Electronics and Information Engineering, Tongji University, Shanghai, November 2010

Ucar S, Ergen S C and Ozkasap O 2013 VMaSC: Vehicular multi-hop algorithm for stable clustering in Vehicular Ad Hoc Networks, Wireless Communications and Networking Conference (WCNC), 2013. IEEE 2381(2386): 7-10

Vodopivec S, Bester J and Kos A 2012 A survey on clustering algorithms for vehicular ad-hoc networks. Telecommunications and Signal Processing (TSP), 2012 35th International Conference 52(56): 3-4

Wang L X 1994 Adaptive Fuzzy systems and control, Prentice Hall, N.J. 